\title{
TRANSPORT MODELING BY MULTI-AGENT SYSTEMS: A SWARM INTELLIGENCE APPROACH
}

\author{
DUŠAN TEODOROVIĆ \\ The Charles E. Via Jr Department of Civil and Environmental Engineering, \\ Virginia Polytechnic Institute and State University, 7054 Haycock Road, Falls \\ Church, VA 22043, USA \\ (Received 2 June 2001; Revised 11 April 2003; In final form 27 August 2003)

\begin{abstract}
There are a number of emergent traffic and transportation phenomena that cannot be analyzed successfully and explained using analytical models. The only way to analyze such phenomena is through the development of models that can simulate behavior of every agent. Agent-based modeling is an approach based on the idea that a system is composed of decentralized individual 'agents' and that each agent interacts with other agents according to localized knowledge. The agent-based approach is a 'bottom-up' approach to modeling where special kinds of artificial agents are created by analogy with social insects. Social insects (including bees, wasps, ants and termites) have lived on Earth for millions of years. Their behavior in nature is primarily characterized by autonomy, distributed functioning and self-organizing capacities. Social insect colonies teach us that very simple individual organisms can form systems capable of performing highly complex tasks by dynamically interacting with each other. On the other hand, a large number of traditional engineering models and algorithms are based on control and centralization. In this article, we try to obtain the answer to the following question: Can we use some principles of natural swarm intelligence in the development of artificial
\end{abstract} \\ systems aimed at solving complex problems in traffic and transportation?
}

Keywords: Transport modeling; Swarm intelligence; Multi-agent systems

\section{INTRODUCTION}

Many traffic and transportation problems show a complex behavioral pattern. We see, for example, urban or air traffic congestion as emergent 
phenomena, which are frequently unpredictable and even sometimes counterintuitive. It is very difficult (if not impossible) to explain the relationships between individual behavior (drivers, passengers, cars, airlines, airports, etc.) and actions and these emergent phenomena they cannot be analyzed successfully and explained using analytical models. The only way to analyze such emergent phenomena is through the development of simulation models that can simulate the behavior of every agent. Agent-based modeling is an approach based on the idea that a system is composed of decentralized individual 'agents' and that each agent interacts with other agents according to their localized knowledge. In our case, the interacting agents might be drivers, passengers, cars, airlines, airports, traffic authorities, and/or government. Kikuchi et al. [1] have pointed out that: 'Transportation activities take place at the intersection between supply and demand in a complex physical, economic, social and political setting. The overall performance of the system reflects the outcome of complex interactions of the individual agents. We feel that Agent-based modeling holds a promise in application to transportation analysis, because this approach is not just a specific computational tool, but a concept and a pattern of thinking'. This agent-based approach to modeling is often called the 'bottom-up' approach.

Special kinds of artificial agents are the agents created by analogy with social insects. Communication systems between individual insects in nature contribute to the formation of the "collective intelligence' of social insect colonies. Recently, the term 'swarm intelligence' has been applied, denoting this 'collective intelligence'. Swarm intelligence (SI) concepts are not currently employed in solving traffic and transportation engineering problems. We strongly believe that SI has the potential to offer many new ideas that could inform a new research approach and novel algorithms to traffic and transportation engineering problems. The first aim of this article is to acquaint the reader with the basic elements of SI, its applications to date in engineering, management and control, and to indicate the directions for future research in this area. The second aim is to show how some principles of natural SI could be used in the development of artificial systems aimed at solving complex problems in traffic and transportation. The organization of the article is as follows: Section 2 explains the basic characteristics of SI; the relationship between SI and multi-agent systems (MAS) is discussed in Section 3; the need for SI approaches in transport modeling are described in Section 4; the basic characteristics of the ant system are explained in Section 5; in Section 
6 , the basic concept of the fuzzy ant system is proposed and Section 7 is devoted to a new computational paradigm - the bee system; potential applications of SI in traffic and transportation are discussed in Section 8; and finally in Section 9 we present our conclusions and recommendations for further research.

\section{WHAT IS SWARM INTELLIGENCE?}

Social insects (including bees, wasps, ants and termites) have lived on Earth for millions of years, building nests and more complex dwellings, organizing production and procuring food. It has also been noted that they care about order and cleanliness, perform funerals, occasionally move around, have a communication and warning system, maintain an army, wage wars and divide labor. In addition, the colonies of social insects are very flexible and can adapt well to the changing environment. There have been examples of 'higher specialization' workers taking on the duties of 'lower specialization' workers when there was a shortage of the latter. This flexibility allows the colony to be robust and maintain its life in spite of considerable disturbances [2].

Interaction between individual insects in a colony of social insects has been well documented. The examples of such interactive behavior are bee dancing during food procuring, ants' pheromone secretion, and performance of specific acts which signal the other insects to start performing the same actions. These communication systems between individual insects contribute to the formation of the "collective intelligence' of social insect colonies. Recently, the term 'swarm intelligence', denoting this 'collective intelligence', has came into use [2-5].

As with honeybees, the self-organization of ants is also based on relatively simple rules of individual insect behavior [6-10]. In the majority of ant species a number of 'scouts' leave the nest foraging for food [11]. Ants successful at finding food leave the pheromone trail behind them so other ants can follow in order to reach food. The appearance of the new ants at the pheromone trail reinforces the pheromone signal. This comprises typical autocatalytic behavior, i.e. the process that reinforces itself and thus converges fast. The 'explosion' in such processes is regulated by a certain limitation mechanism. In the ant case, the pheromone trail evaporates with time. In this behavioral pattern the decision of an ant to follow a certain path to food depends on the behavior of its nestmates. At the same time, the 
ant in question also increases the chance that the nestmates leaving the nest after it will follow the same path. In other words, one ant's movement is highly determined by the movement of previous ants.

Self-organization of bees is based on a few relatively simple rules of individual insect behavior [12-25]. In spite of the existence of a large number of different social insect species, and variation in their behavioral patterns, it is possible to describe individual insects' behavior as follows [26]: Each bee decides to reach the nectar source by following a nestmate who has already discovered a patch of flowers. Each hive has a so-called dance floor area in which the bees that have discovered nectar sources dance, in that way trying to convince their nestmates to follow them. If a bee decides to leave the hive to get nectar, it follows one of the bee dancers to one of the nectar areas. Upon arrival, the foraging bee takes a load of nectar and returns to the hive relinquishing the nectar to a food storer bee. After the food is relinquished, the bee can: (a) abandon the food source and become again an uncommitted follower; (b) continue to forage at the food source without recruiting nestmates; or (c) dance and thus recruit the nestmates before the return to the food source. The bee opts for one of the above alternatives with a certain probability. Within the dance area the bee dancers 'advertise' different food areas. The mechanisms by which the bee decides to follow a specific dancer are not well understood, but it is considered that the recruitment among bees is always a function of the quality of the food source [26]. It is also noted that not all bees start foraging simultaneously. Experiments confirmed that new bees begin foraging at a rate proportional to the difference between the eventual total and the number presently foraging.

Interaction and self-organization are also present in many transportation phenomena. Let us consider, for example, the route choice problem. Do we somehow 'collaborate' with other drivers when making a route choice? Are we 'in conflict' with other drivers when making a route choice? The more drivers choose a certain route, the lower the probability the 'incoming' ones will do the same. The higher the congestion on a particular link, the less likely it is for an arriving driver to choose that link. It is important not to forget that congestion is a consequence of many decisions different drivers make. In other words, drivers who choose a specific route before we do, influence our route choice decision to some extent. In the same way, we have a certain influence on the route choice of drivers that will make the decision after us. Let us look at another example. When a traffic incident causes two lanes on a highway to be relatively congested, and 
the third one stays with relatively low density, a number of drivers will try to change lane and enter the 'fast' one. When we realize such behavior is successful we start to consider entering the 'fast lane' and potentially decrease our travel time. That is, 'success' in decreasing travel time of other drivers indirectly tells us something like "come and join us', 'do the same', etc. This kind of behavior is very similar to the bee dancing during food procuring. By dancing, bees are trying to recruit their nestmates for the good nectar source. Showing that we can significantly decrease our travel time, the other drivers are recruiting us for the 'fast lane' on the highway. This kind of positive feedback will undoubtedly decrease total travel time for a certain number of drivers. At a specific moment, drivers could start competing to enter the 'fast lane'. This will finally cause congestion in the 'fast lane'. In other words, competition and saturation represent a type of negative feedback. The more bees fly to a 'good' nectar source, the more that source becomes congested and less rich in nectar. Positive and negative feedback are obviously very important characteristics of swarm behavior phenomena. Let us consider a third example. During winter, students always find the 'best path' through the snow on campus. In the greatest number of cases, the 'new' student will follow the existing path, at the same time making that path even more visible for incoming pedestrians. This is very similar to the behavior of ants successful at finding food, that leave the pheromone trail behind them, so other ants can follow in order to reach food. Human beings are the decisionmakers within different traffic and transportation systems (pedestrians, drivers, passengers, etc.). Human beings have been on the Moon, created the Mona Lisa, wrote Romeo and Juliet and invented the Internet, but will still very often join other drivers and park their cars illegally in a forbidden area, as members of a 'driver swarm'.

It should be noted that a large number of traditional engineering models and algorithms are based on control and centralization. On the other hand, bee or ant swarm behavior in nature is primarily characterized by autonomy and distributed functioning and self-organizing. Social insect colonies teach us that very simple individual organisms can form systems capable of performing highly complex tasks by dynamically interacting with each other. It is of course of great importance to investigate both advantages and disadvantages of autonomy, distributed functioning and self-organizing in relation to traditional engineering methods that rely on control and centralization. The basic question about the above-mentioned characteristics of social insects that should be answered is: Can we use some principles of 
natural SI in the development of artificial systems aimed at solving complex problems in traffic and transportation?

It is important to state here that the development of artificial systems does not entail the complete imitation of natural systems, but explores them in search of ideas and models. The successful application of emergent techniques based on natural metaphors (e.g. vehicle fleet planning and static and dynamic routing and scheduling of vehicles and crews for airlines, railroads, truck operations and public transportation services, designing transportation networks and optimizing alignments for highways and public transportation routes through complex geographic spaces, different locations problems, etc.), such as simulated annealing, genetic algorithms and neural networks, to complex transportation engineering problems are certainly encouraging. They most definitely point to natural systems as a source of ideas and models for development of various artificial systems.

\section{SWARM INTELLIGENCE AS A PART OF MULTI-AGENT SYSTEMS}

SI is a part of artificial intelligence (AI). In practice, the main aim of AI during the last four decades has been to develop 'intelligent machines' with the capabilities for solving complex tasks similar to human beings. AI has passed through a number of different phases in the past. A very important area of AI is expert systems, computer programs based on expert knowledge and capable of performing complex tasks to a certain extent. When trying to develop very complex expert systems, researchers realized it is rather difficult to discover and detect 'unique knowledge' and to incorporate it into a knowledge base. When solving problems in everyday life, or in engineering practice, we collaborate with others, we learn from others, we make different adjustments, we 'fight' for our goals, we sometimes negotiate, and all the time we exchange some information and knowledge. Biologists, psychologists and many other scientists contributed significantly to the area of AI. Engineers who are trying to apply some of the AI techniques have to agree with biologists, psychologists and sociologists that intelligence cannot be considered as a purely individual attribute without any social context. The 'classic' AI and approaches based on principles of centralization and sequential computing should be carefully explored and compared with the approaches based on problem solving by individual entities. These 
entities or agents could communicate and cooperate among themselves, exchange information and try to solve complex problems together. MAS can be described as systems composed of physical individuals (robots, for example) or 'virtual' (artificial) ones that communicate among themselves, cooperate, collaborate, exchange information and knowledge and perform some tasks in their environment. In other words, scientists have enormous freedom to create different MAS, defining the environment, as well as different artificial autonomous entities that live in such an environment. Success in solving a particular class of complex problem is the only criterion for evaluating a specific MAS. Distributed AI represents a relatively new area of AI, therefore all of its potential advantages and disadvantages should be explored in great detail. MAS can be composed of 'intelligent' agents or 'reactive' agents. An 'intelligent' agent has some level of intelligence, knowledge, certain skills and can communicate, collaborate or 'fight' with other agents. Usually, an 'intelligent' agent has certain goals it wants to achieve. 'Reactive' agents are not 'intelligent'. They can interact directly or indirectly with other agents, and they 'react' to different events or communication signals from other agents. However, they are autonomous. These communication systems between individual 'reactive agents' contribute to the formation of the 'collective intelligence' of the whole MAS. Bees, wasps or ants are 'reactive' agents, since bee dancing, ants' pheromone secretion, or performing another act, indicate and signal to the other insects to start performing a consecutive action. The term 'swarm intelligence' can be related to the MAS composed of 'reactive' agents. Once more, it is important to say that the development of artificial systems does not entail the complete imitation of natural systems. Natural systems should be studied primarily for ideas, models and analogies. Therefore, strict and rigid classifications, used to describe 'intelligent' and 'reactive' agents, should not be perceived literally. As researchers trying to solve a complex problem, we have considerable liberty to create a completely 'artificial world', thus an 'artificial environment with artificial life', with the agents that are 'more or less' 'intelligent' or more or less 'reactive'.

\section{A SWARM INTELLIGENCE (MULTI-AGENT SYSTEM) APPROACH TO TRANSPORT MODELING}

Physically distributed systems, their combinatorial nature, multiple 
conflicting criteria, uncertainty (randomness, stochasticity, fuzziness) and time-dependence (dynamic, real-time) are some of the attributes characterizing the complexity of contemporary traffic and transportation engineering problems. Let us discuss these attributes in more detail.

All transportation systems are physically distributed systems. Significant numbers of trips are made using urban and intercity transport, and thousands tonnes of freight are transported every day. When traveling, passengers and/or drivers are making certain decisions. These decisions are related to choice problems including, for example, choosing the mode of transportation, choosing the class in the aircraft, choosing the public transportation route, choosing the route to drive, choosing departure time, etc. Who could be considered as an agent ('artificial bee', 'artificial ant') in such transportation systems? For instance, all passengers could be thought of as agents. In some other situations, all drivers could be agents. If we investigate problems of avoiding collisions in air traffic control or those related to air traffic control system congestion, all aircraft could be considered agents. In other words, when modeling transport phenomena, depending on the problem's context, agents could be passengers, dispatchers, drivers, operators, air traffic controllers, vehicles, aircraft, vessels, terminals, intersections or whatever. Sometimes agents cooperate and at other times they are in conflict. Increasing numbers of cars on streets and aircraft in airspace create conflict situations. On the other hand, drivers obeying traffic light instructions and pilots who strictly follow air traffic control rules are cooperative agents.

It is important to emphasize that sometimes a 'bee', an 'ant', or an agent can be a completely artificial entity which we create in order to solve a particular, complex transportation problem. In other words, transportation networks, pedestrians, drivers, dispatchers, pilots, vessels, aircraft or cars do exist as real entities, but we introduce artificial entities - agents - into the analysis, as a computational tool, to help us solve the problem.

Most complex transportation engineering problems are combinatorial by their nature. A large portion of social insects' activities is tied to food foraging. It is known that honeybees normally spend the last part of their life collecting food [27]; they also spend a considerable portion of their life span learning and improving their foraging skills [19]. Every bee colony has scouts who are the colony's explorers [28]. The explorers do not have any guidance while looking for food. They are concerned primarily with finding any kind of food source. As a 
result of such behavior, the scouts are characterized by low search costs and a low average in food source quality. Occasionally, the scouts can accidentally discover rich, entirely unknown food sources. The artificial scouts attempting to solve difficult combinatorial optimization problems could have as a task the fast discovery of a group of feasible solutions. Some of those feasible solutions to the difficult combinatorial optimization problems could then prove to be solutions of very good quality. In the case of honeybees, the recruitment rate represents a 'measure' of how quickly the bee colony finds and exploits a newly discovered food source. Artificial recruiting could similarly represent the 'measurement' of the speed with which the feasible solution or the 'good quality' solution is found. The cooperation between the insects decreases foragers' costs in finding new food sources. This suggests that cooperation between artificial agents would also allow for the fast discovery of the feasible solution. It is also known that cooperation increases the quality of the food sources located by foragers. This implies that cooperation could also help us find the best solutions to difficult combinatorial optimization problems. The survival and progress of the bee colony is dependent upon rapid discovery and efficient utilization of the best food resources. In other words, the successful solution of difficult engineering problems (especially those that need to be solved in real time) is connected to the relatively fast discovery of 'good solutions'.

A number of complex transportation engineering problems are also characterized by multiple conflicting criteria. Fleet size and total transportation costs conflict with the level of service provided to passengers. When matching transportation demand and supply, these conflict criteria and interests should certainly be taken into account. Could we develop MAS consisting of a few different agent types, each one representing a particular interest group (passengers, carriers, vehicles)? For instance, drivers are in conflict among themselves since they have to share limited resources (road capacity); or, aircraft are in conflict because of limited runway and airspace sector capacities. Could we develop MAS composed of the same agent types (competitor agents) who would fight among themselves for limited resources?

Uncertainty is another common characteristic of many complex transportation problems. Uncertainty also significantly influences the real, every day life of ant and bee colonies. Social insects look continuously for food primarily by performing random and semirandom searches. Discovering good food sources is a complex task portrayed by uncertainty. Development of MAS, which would solve 
the problem of uncertainty in transportation systems, is a highly important research task. Agents in such systems would use approximate reasoning and rules of fuzzy logic in their communication and acting.

Numerous complex transportation problems are characterized by time dependence. Flows in transportation systems vary over time. At the same time, many transportation phenomena could also be described by their autocatalytic behavior. In essence, many processes in transportation systems reinforce themselves and converge fast. The appearance of more new drivers on a faster route over some time interval reinforces the 'signal' to other drivers and decreases the probability of choosing the route in question. A driver's decisions to choose a certain route is time dependent and depends highly on other drivers' behavior.

A SI approach could be applied to a variety of traffic and transportation engineering problems, including: vehicle and crew routing and scheduling for airlines, rail systems, public transportation, discrete location problems (hub locations and emergency facility locations), supply chain management, highway alignment, designing transportation networks, transportation planning (route choice, traffic assignment and user and system equilibrium), air traffic control, or urban traffic control. Modern transportation networks are very large, characterized by complex relationships between particular nodes and links, and frequently congested. Therefore, it is quite difficult to monitor and analyze them to find appropriate solutions for problems, and probably impossible to control the whole network in a centralized way. It is therefore self-evident that research efforts would be beneficial in order to achieve decentralized monitoring, analysis and control. To be more specific, 'bees' or 'ants' would be at the nodes or links, trying to solve problems as autonomous agents at the local level, at the same time cooperating among themselves. This approach could eventually lead to a discovery of good solutions for the problems related to the whole transportation network. In years to come, research in the area of SI applications in traffic and transportation could help us find the answers to the following questions:

- What is the real potential of SI in traffic and transportation research?

- Can we use some principles of natural SI in the development of artificial systems aimed at solving complex problems in transportation engineering?

- Should artificial ants, termites or bees (agents) be regarded as equal, 
or should there be several types of agent? Should they have the ability to learn? Should they communicate directly or indirectly? In other words, we need to ask what kind of system performance various types of dynamic interactions between different system elements could achieve.

- When should principles of autonomy, decentralization, selforganizing, flexibility and robustness be used when developing artificial systems based on the analogy of social insect colonies?

- What could be the potential of artificial SI systems in traffic and transportation? For what type of problems is artificial SI most useful?

- Are there any possibilities for the hybrid combination of SI and other artificial intelligence approaches and different heuristic algorithms?

- Could SI be combined with other techniques (e.g. fuzzy set theory) to solve complex traffic and transportation engineering problems characterized by uncertainty?

- What are the costs and benefits of the development of artificial systems based on natural SI in transportation engineering?

\section{ANT SYSTEM}

The most important result of artificial system development based on SI is the creation of the ant system and the ant colony system [2, 29-34]. The ant colony system is a new metaheuristic for hard combinatorial optimization problems. Dorigo et al. [30] applied the ant system to the classic traveling salesman problem. They also tested their approach on the asymmetric traveling salesman problem, the quadratic assignment problem and the job-shop scheduling problem. Bullnheimer et al. [35, 36] used the ant system to solve the vehicle routing problem in its basic form (i.e. homogenous fleet, capacity restriction, distance restriction, one central depot) and obtained very good results.

Dorigo et al. [30] introduced the ant system in trying to solve the traveling salesman problem. Given a set $N$ of $n$ towns (nodes), this well-known problem is based on finding the shortest route the traveling salesman should take when visiting each town exactly once. The traveling salesman departs from and returns to a depot located in one of the towns.

Artificial ants, proposed by Dorigo et al. [30], search the solution space, simulating real ants looking for food in the environment. The 
objective function values correspond to the quality of food sources. Existence of an adaptive memory characterizes the searching process. In our case, the adaptive memory corresponds to the pheromone trials. It should be emphasized that time in the artificial ants' environment is discrete. At the beginning of the search (time $t=0$ ), ants are located in different towns. Dorigo et al. [29] denoted by $\tau_{i j}(t)$ the intensity of trial on edge $(i, j)$ at time $t$. In time point $t=0$ the value $\tau_{i j}(0)$ is equal to a small positive constant $c$. At time $t$, every ant is moving from the current town to the next one. Reaching the following town at time $(t+1)$, every ant moves towards a 'new' (as yet unvisited) town. Located in town $i$, ant $k$ chooses to visit the next town $j$ at time $t$, with the transition probability $p_{i j}^{k}(t)$ defined by the following relation:

$$
p_{i j}^{k}(t)=\left\{\begin{array}{lr}
\frac{\left[\tau_{i j}(t)\right]^{\alpha}\left[\eta_{i j}\right]^{\beta}}{\sum_{h \in \Omega_{j}^{k}(t)}\left[\tau_{i h}(t)\right]^{\alpha}\left[\eta_{i h}\right]^{\beta}}, & \text { if } j \in \Omega_{i}^{k}(t) \\
0, & \text { otherwise }
\end{array}\right.
$$

where $\Omega_{i}^{k}(t)=$ set of feasible nodes to be visited by ant $k$ (the set of feasible nodes is updated for each ant after every move); $\mathrm{d}_{i j}=$ Euclidean distance between node $i$ and node $j ; \eta_{i j}=1 / d_{i j}$ 'visibility'; and $\alpha, \beta=$ parameters representing the relative importance of trail intensity and visibility.

The greater the importance the analyst gives to visibility, the greater the probability that the nearest towns will be selected. When trial intensity is given greater importance, the highly desirable links will be the ones through which many ants have already previously passed.

Dorigo et al. [30] defined iteration as $m$ moves performed by $m$ ants in the time interval $(t, t+1)$. After $\mathrm{n}$ iterations every ant will have completed the traveling salesman tour. Dorigo et al. [30] also stated that $n$ iterations represent a cycle. They proposed that trial intensity should be updated after every cycle in the following way:

$$
\tau_{i j}(t+n)=\rho \tau_{i j}(t)+\Delta \tau_{i j}
$$

where: $\rho=$ the coefficient $(\rho<1)$ such that $(1-\rho)$ represents evaporation of the trial between time $t$ and $t+n$.

The total increase in trial intensity along link $(i, j)$ after one completed cycle is equal to: 


$$
\Delta \tau_{i j}=\sum_{k=1}^{m} \Delta_{i j}^{k}
$$

where $\Delta_{i j}^{k}$ is the quantity of pheromone laid on link $(i, j)$ by the $k$ th ant during time interval $(t, t+n)$.

The quantity $\Delta_{i j}^{k}$ is given by:

$\Delta_{i j}^{k}(t)= \begin{cases}\frac{Q}{L_{k}(t)}, & \text { if the } k \text {-th ant walks along the link }(i, j) \text { in its tour } \\ 0, & \text { otherwise }\end{cases}$ where $Q=$ a constant; and $L_{\mathrm{k}}(t)=$ the tour length developed by the $k$ th ant within the cycle.

\section{FUZZY ANT SYSTEM}

Among others, Lučić and Teodorović [37] and Teodorović and Lučić [38] made an attempt to modify the 'classical' ant system. They proposed the fuzzy ant system (FAS). The basic modification would be in the way of calculating transition probabilities, so that fuzzy logic is used. When using fuzzy logic as a separate module within the ant system, it is possible to deal with the uncertainty which sometimes exists in complex combinatorial optimization problems. The control strategies of the ant can also be formulated in terms of numerous descriptive rules. Zadeh [39] first introduced the original idea of the possibility of decision-making based on imprecise, qualitative data by combining descriptive linguistic rules through fuzzy logic. The combination of imprecise logic rules in a single control strategy is called by Zadeh [40] approximate or fuzzy reasoning. Zadeh [39, 40] first presented fuzzy logic as a means of processing vague, linguistic information. Fuzzy rules include descriptive expressions such as small, medium, or large used to categorize the linguistic (fuzzy) input and output variables. A set of fuzzy rules, describing the control strategy, forms a fuzzy control algorithm, that is, approximate reasoning algorithm, whereas the linguistic expressions are represented and quantified by fuzzy sets. The main advantage of this approach is the possibility of introducing and using rules from experience, intuition and heuristics, and the fact that a model of the process is not required. Fuzzy reasoning (approximate reasoning) involves the transformation of a group of fuzzy rules into fuzzy relations in order to achieve a result. Fuzzy reasoning is an inference procedure, therefore the way of 
generating the conclusion from the premises when the linguistic expressions are quantified by fuzzy sets. The inference engine of the fuzzy logic system maps fuzzy relations into fuzzy sets. The inference engine handles the way in which rules are combined [41]. There are a number of inferential procedures in the literature.

When making a decision about the next node to be visited, an ant takes both 'visibility' and pheromone trial intensity into consideration. We can assume that an ant can perceive a particular distance between nodes as 'small', 'medium' or 'large', and the trial intensity as 'weak', 'medium' or 'strong'. Depending on the distance from the next node, as well as the trial intensity, the ant chooses the next link with the greater or lesser probability. These probabilities can be described by appropriate fuzzy sets.

An approximate reasoning algorithm for calculating the probability of choosing the next link could consist of rules of the following type:

If distance is SMALL and trial intensity is STRONG

Then probability is VERY HIGH

The approximate reasoning algorithm could replace the original relation for calculating transition probabilities. Therefore, it would be possible to calculate transition probabilities even if some of the input data were only known approximately. It seems that a FAS could be a very powerful tool in solving different transportation engineering problems characterized by their combinatorial nature and uncertainty. The author's preliminary results with the FAS are promising.

\section{BEE SYSTEM: A NEW COMPUTATIONAL PARADIGM}

In their preliminary research, Lučić and Teodorović [37, 42, 43] have developed the bee system that represents the new concept within the area of SI. This bee system is heavily inspired by the analogy of bee behavior. The main goal of Lučić and Teodorović $[37,42,43]$ research was not to develop a new heuristic algorithm for the traveling salesman problem but to explore possible applications of SI (particularly collective bee intelligence) in solving complex engineering and control problems. The traveling salesman problem is only an illustrative example, which shows the characteristics of the proposed concept.

The bee system was tested in preliminary research on a number of numerical examples. Benchmark problems were taken from the University of Heidelberg's Internet site < http://www.iwr.uni-heidelberg. 
de/iwr/comopt/software/TSPLIB95/tsp/ $>$. The problems considered were: Eil51.tsp, Berlin52.tsp, St70.tsp, Pr76.tsp, Kroa100.tsp, Eil101.tsp, A280.tsp, Pcb442.tsp and Pr1002.tsp. (All tests were run using a PC with PIII processor, 533MHz.) The results obtained are given in Table I.

As we can see from Table I, the bee system offers high quality results. Computer times required for finding out the best solutions were very low. In other words, the bee system was able to provide 'very good' solutions in a 'reasonable amount' of computer time.

\section{SOME POTENTIAL APPLICATIONS OF SWARM INTELLIGENCE IN TRAFFIC AND TRANSPORTATION}

This section describes some of the potential applications of SI in traffic and transportation engineering. The following examples were chosen from a large number of potential applications primarily for illustrative purposes.

The transit network-planning problem [44] can be defined as follows: for known estimated annual numbers of passengers between individual pairs of nodes, the shape of a network of public transportation lines and their corresponding service frequencies should be determined, taking into account operator revenues and costs as well as the level of service offered to passengers. The problem of determining the shape of public transportation routes and corresponding service frequencies is combinatorial by its nature. It should also be emphasized that when public transportation is established among a large number of nodes, the dimensions of the problem become enormous. Transit network planning and design is an ideal problem for a SI application. It would be very interesting to see how successful 'ants' or 'bees' are in transit network design.

Timetabling and schedule synchronization [38] are the planning phases that follow transit network design, detailed line alignment, frequency determination and line headways. In other words, schedule synchronization can be achieved only after determining detailed line alignment and line headways. Trips between any two nodes in a public transit network may or may not include a transfer. A direct connection could be provided only to a certain number of passengers, primarily for economic reasons. At the same time, some passengers use different modes of transportation (e.g. bus, light rail, tram, metro, trolley bus, coordinated dial-a-ride, etc.). Although they represent inconvenience to 


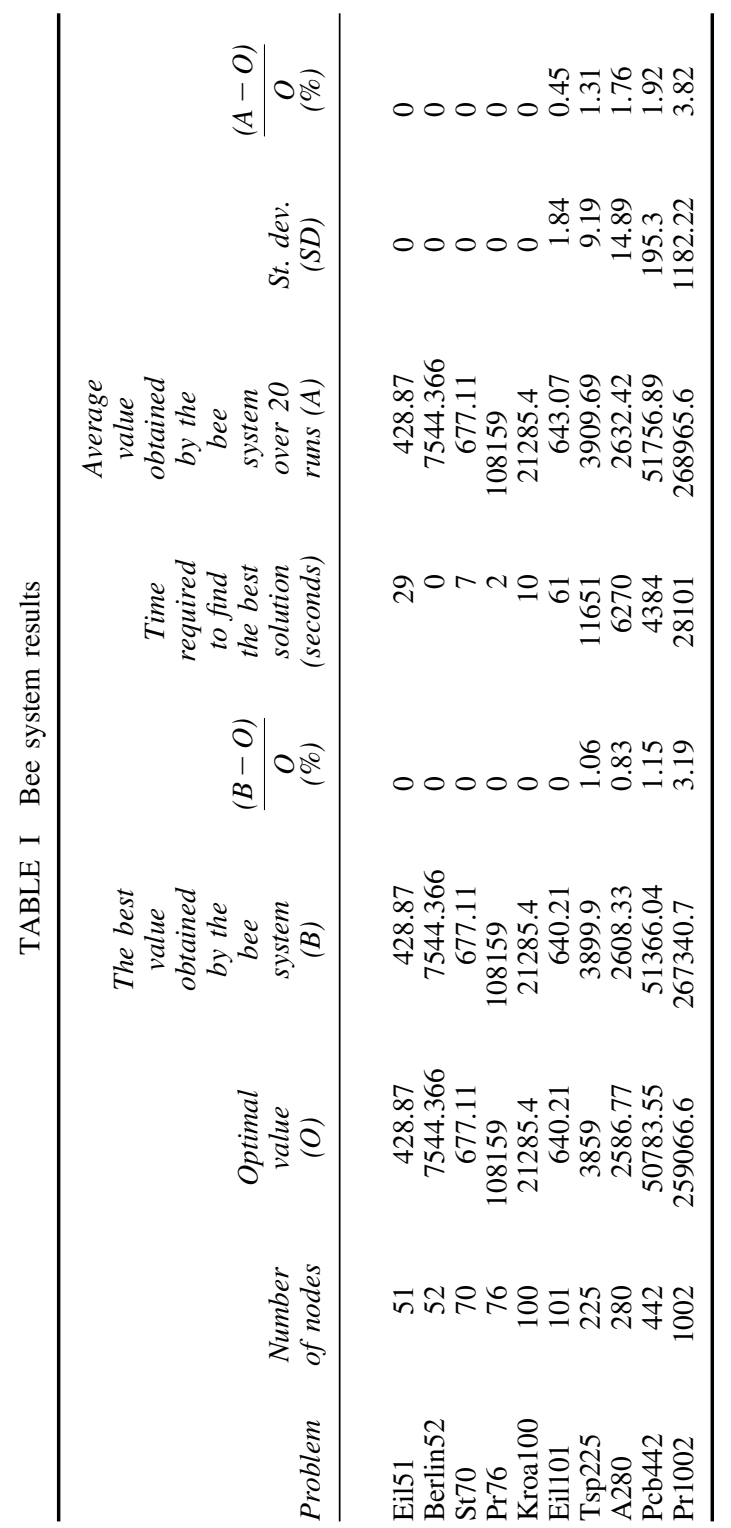


passengers, transfers cannot be completely avoided in public transit. It is especially important to carefully synchronize schedules in cases of larger headways, since badly coordinated transfers significantly increase waiting times. At the same time, poorly coordinated transfers could also decrease the total number of passengers using public transit and their switching to other competitive modes (especially private cars). Optimal schedule synchronization assumes minimal total waiting times of all passengers at transfer nodes. Real world instances of the schedule synchronization problem could be tremendous.

The alignment problem for public transportation routes assumes selection of the 'best' path to connect two points on a map. The 'best' alignment $[45,46]$ minimizes total costs and satisfies the engineering design, operational constraints and established level of service. This a difficult combinatorial optimization problem which could be characterized by uncertainty, as well as the existence of a few usually conflicting criteria. Operator's economic results, as well as the level of service offered to passengers, highly depend on the alignment of public transportation routes. Development of hybrid models (ant system with fuzzy logic, or bee system with fuzzy logic) for solving alignment problems would be of great benefit for public transportation companies and the broader traveling public.

Every vehicle trip in public transportation is characterized by an origin (terminal at the beginning of a transit line), destination (terminal at the end of a transit line), departure time, expected duration of the trip and time points at different stops along the line. The sequence of trips is known as a block. Since in most cases buses leave the depot more than once a day, they usually perform more than one block per day. The generic version of the vehicle-scheduling problem can be defined in the following way: assign the minimum possible number of vehicles for planned trips so that all trips are performed by the vehicles located at a single depot and total operational costs are minimized. The generic vehicle-scheduling problem can be transformed into different versions.

Labor costs in the transit industry are very high. This means that the run cutting (crew scheduling) problem must be studied very carefully taking into account operator's as well as crew's objectives [47, 48]. Once the vehicle schedule has been designed, scheduling crews and their assignment to planned duties are among the most important problems transit operators face. The run-cutting (crew scheduling) problem is a very complex one and it is characterized by the following factors: (a) large dimensions (many transit lines, buses and operators); 
(b) variability in demand for crews during the day (caused by variability in passenger travel demand during the day, corresponding variability in the number of trips to be performed and variability in the number of buses needed); and (c) complex operator labor agreements (the rules regulating crew operations). When performing their duty, crews change vehicles from time to time. The segment of a crew schedule during which it is continually in the same vehicle is usually called a piece. One possible combination of pieces is called the run (shift). By assigning a particular run to a crew, its workday is defined completely. The run cutting (crew scheduling) problem can be formulated as follows: for a given vehicle schedule, schedule the crew 'in the best possible way', while simultaneously respecting all the rules that regulate crew operations.

Dial-a-ride [49] is a type of paratransit service that provides shared ride public transportation for citizens with disabilities, seniors, or the general public. Different versions of the dial-a-ride problem are found in every day practice - for example: transportation of people in low-density areas, transportation of the handicapped and elderly, and parcel pick-up and delivery services in urban areas. A carrier that provides the service receives inquiries for transportation. Origin, destination and preferred start time of the transportation service characterize every request. The carrier's 'job' is to design vehicle routes and schedules of vehicles to optimize objectives such as maximizing passenger trips served, minimizing the total traveling distance, reducing the number of vehicles needed, and minimizing detour time. In the static version of the dial-a-ride problem, it is customary to collect requests for transportation a day prior to the beginning of service. All customers demanding service define pick-up and delivery location, as well as preferred beginning of the service. In the case of the dynamic dial-a-ride system all routing and scheduling decisions must be done in real time. Police cars, ambulances and taxi dispatching are also situations that require real time dispatching decisions. A logical question which arises is: Who should respond to a new request? It is obvious that all requests must be divided among available units in a way that provides maximum efficiency of the whole system. Labor division is one of the main traits of the social insects colonies. Assigning vehicles to planned trips, defining crew workday in public transportation, or allocating available units to the transportation or emergency request are good examples of transportation problems related to labor division.

Vehicle and crew routing and scheduling problems appear in various 
transportation activities. In the case of distribution systems, vehicles usually leave the depot, serve the nodes in the network and return to the depot on completion of their routes. Every node is described by a certain demand (the amount to be delivered to the node or the amount to be picked up from the node). Other known values include the coordinates of the depot and nodes, the distance between all pairs of nodes, and the capacity of the vehicles providing service. The classical vehicle routing problem consists of finding the set of routes that minimize transport costs. Further variations of this problem include existence of few depots in the network, performing the service with several types of different vehicles, uncertain demand at nodes, or existence of the time windows for carrying out the service at certain nodes. Development of hybrid models (bee system, fuzzy logic and visualization) for solving complex vehicle routing and scheduling problems would be of great benefit for both distribution companies and the general public.

Designing an airline or utility network is an extremely complex planning task, combinatorial in its nature. Network shape and flight frequency on individual links directly affect operator's business results and the quality of service provided to passengers. Designing the best hub-and-spoke architecture represents a typical example of the facilities location problem. Packages or passengers in intercity transport are very frequently routed through one or more hubs, on their way from origin to destination. In other words, instead of routing passengers from each origin directly to their destination, the hub-and-spoke system transports passengers and packages through hubs. Determining the best architecture of the hub-and-spoke system has great significance. The highly competitive environment in air transportation, logistics and communication assumes the best possible hub-and-spoke architecture. A major goal of this research would be introducing swarm intelligence and its application to generic air transportation network design, as well as to the generic hub-and-spoke design problem. Potential sources of possible ideas could include studying the brood sorting or cemetery organization in social insect colonies.

In recent years, congestion has appeared in numerous parts of the air traffic control system (attributable to many airports, numerous air routes and air traffic control sectors). Congestion arises due to the fact that in certain time periods, 'demand' at an airport or on an air route exceeds its capacity. Major airports usually have multiple runways in operation. Assignment of aircraft mix and take-off and landing operations could be accomplished in many different ways. Every possible 
runway-operating configuration has its appropriate capacity value. Previous research and every day practice has revealed and proved that landing capacities are the main cause of aircraft delays. During the last decade, airport development has not been compatible with an increasing demand for that airport. This is one of the main causes of congestion. Congestion is also highly influenced by the fact that in air traffic control, demand for airports as well as airport capacities are not constant. Arrival times of longer-haul flights are quite often changed because of meteorological conditions. Maintenance problems, aircrew absenteeism, meteorological conditions or airport equipment failure may cause some flights to be delayed or cancelled. The variations in airport capacities could be even greater. The most frequent congestion occurs at airports, where meteorological conditions (such as ceiling, visibility and wind velocity and direction) have a direct influence on airport capacity. Since precise forecasts of meteorological conditions is not possible over a long period of time, the capacity of an airport can be only approximately determined over the long run. Different actions could be undertaken in order to minimize negative consequences of congestion. Air traffic flow management represents a set of actions performed to prevent overloading of any element of the air traffic control system, to provide maximum utilization of existing capacities, to minimize airline delay costs and passenger inconvenience. The basic goal of this research would be to introduce swarm intelligence and its potential application to a generic air traffic flow management problem.

Many models have been developed for dealing with transportation problems during past four decades. Most frequently, the basic assumption in these models was that all planned transportation activities would be performed without any disturbances. Every day, certain buses have a delay, some of the planned flights are cancelled, traffic incidents occur on highways, some links are completely congested, and meteorological conditions cause lower traffic flow speeds in particular locations. How will an artificial system design based on swarm intelligence principles behave in unusual, unpredictable situations? Will it be able to find reasonable 'good' solutions for the complex transportation problems caused by unpredictable events? Will the autonomy, distributed functioning and self-organizing capabilities of the artificial system significantly help to mitigate disturbances within the transportation system under consideration? Finding the appropriate answers to these questions is without a doubt an important and challenging scientific task. 


\section{CONCLUSION}

The initial applications of the ant and bee systems to some of the difficult transportation engineering combinatorial optimization problems are very encouraging, since they confirm the value of natural systems as a source of ideas and models for the development of a variety of useful artificial systems. SI has already been applied (the ant system) in some other engineering areas such as robotics and the results obtained have been very good.

Bee or ant swarm behavior in nature is primarily characterized by autonomy, distributed functioning and self-organizing capacities. Social insect colonies teach us that very simple individual organisms can form systems capable of performing highly complex tasks by dynamically interacting with each other. It seems that the development of MAS composed primarily of 'reactive agents' would lead to the achievement of reasonably good research results in solving certain transportation problems. In such a way we can avoid problems regarding the development of agents' knowledge base, as well as problems regarding type of 'direct' communication among 'intelligent' agents. It is certainly of great importance to investigate both advantages and disadvantages of autonomy, distributed functioning and selforganizing capacities in relation to traditional engineering methods relying on control and centralization.

This article could be considered as one author's educated guess (based on intuition and prior knowledge) that the SI approach is valid and promising. It is important to remember, however, that even biologists have only partial knowledge about bee or ants behavior in nature. They also need to make some 'educated guesses' in order to explain properly natural SI.

Preliminary results have shown that the development of new models based on SI principles (autonomy, distributed functioning, selforganizing, potential adaptability and robustness) could probably contribute significantly to solving complex transportation engineering problems.

\section{Acknowledgments}

The author wishes to express his gratitude to the US National Science Foundation which has supported this research - grant \# CMS0085430. The author would also like to thank Professor Paul Schon- 
feld of the University of Maryland for his valuable comments, opinions and discussions.

\section{References}

[1] Kikuchi, S., Rhee, J. and Teodorović, D. (2002) 'Applicability of an agent-based modeling concept to modeling of transportation phenomena', YUJOR 12, 141156.

[2] Bonabeau, E., Dorigo, M. and Theraulaz, G. (1999) Swarm Intelligence (Oxford, Oxford University Press).

[3] Beni, G. (1998) 'The concept of cellular robotic system'. In: Proceedings 1988 IEEE International Symposium on Intelligent Control, pp. 57-62 (Los Alamitos, CA, IEEE Computer Society Press).

[4] Beni, G. and Wang, J. (1989) 'Swarm intelligence'. In: Proceedings Seventh Annual Meeting of the Robotics Society of Japan, pp. 425-428 (Tokyo, RSJ Press).

[5] Beni, G. and Hackwood, S. (1992) 'Stationary waves in cyclic swarms'. In: Proceedings 1992 International Symposium on Intelligent Control, pp. 234-242 (Los Alamitos, CA, IEEE Computer Society Press).

[6] Deneubourg, J.L., Aron, S., Goss, S. and Pasteels, J.M. (1990) 'The self-organizing exploratory pattern of the argentine ant', Journal of Insect Behavior 3, $159-168$.

[7] Franks, N.R., Wilby, A., Silverman, B.W. and Tofts, C. (1992) 'Self-organizing nest construction in ants: sophisticated building by blind buldozing', Animal Behavior 44, 357-375.

[8] Beckers, R., Deneubourg, J.L. and Goss, S. (1992) 'Trails and U-turns in the selection of a path by the ant lasius niger', Journal of Theoretical Biology 159, $397-415$.

[9] Nonacs, P. and Soriano, J.L. (1998) 'Patch sampling behavior and future foraging expectations in argentine ants', Linepithema humile, Animal Behavior 55, 519527.

[10] Vienne, K., Erard, C. and Lenoir, A. (1998) 'Influence of the queen on worker behavior and queen recognition behavior in ants', Ethology 104, 431-446.

[11] Deneubourg, J.L., Goss, S., Franks, N. and Pasteels, J.M. (1989) 'The blind leading the blind: modeling chemically mediated army ant raid patterns', Journal of Insect Behavior 2, 719-725.

[12] Gould, J.L. (1987) 'Landmark learning by honeybees', Animal Behavior 35, $26-34$.

[13] Hill, P.S., Wells, P.H. and Wells, H. (1997) 'Spontaneous flower constancy and learning in honeybees as a function of color', Animal Behavior 54, 615-627.

[14] Baschbach, V.S. and Waddington, K.D. (1994) 'Risk-sensitive foraging in honey bees: no consensus among individuals and no effect of colony honey stores', Animal Behavior, 47, 933-941.

[15] Dukas, R. and Real, L.A. (1991) 'Learning foraging by bees: a comparison between social and solitary species', Animal Behavior 42, 269-276.

[16] Kadmoon, R. and Shmida, A. (1992) 'Departure rules used by bees foraging for nectar: a field test', Evolutionary Ecology 6, 142-151.

[17] Peleg, B., Shmida, A. and Ellner, S. (1992) 'Foraging graphs: constraint rules on matching between bees and flowers in a two-sided pollination market', Journal of Theoretical Biology 157, 191-201.

[18] Seeley, T.D. (1992) 'The tremble dance of the honey bee: message and meanings', Behavior Ecology Sociobiology 31, 375-383. 
[19] Dukas, R. and Visscher, P.K. (1994) 'Lifetime learning by foraging honey bees', Animal Behavior 48, 1007-1012.

[20] Keasar, T., Shmida, A. and Motro, U. (1996) 'Innate movement rules in foraging bees: flight distances are affected by recent rewards and are correlated with choice of flower type', Behavior Ecology Sociobiology 39, 381-388.

[21] Chittka, L., Gumbert, A. and Kunze, J. (1997) 'Foraging dynamics of bumble bees: correlates of movements within and between plant species', Behavioral Ecology 8, 239-249.

[22] Chittka, L. and Thompson, J.D. (1997) 'Sensori-motor learning and its relevance for task specialization in bumble bees', Behavior Ecology Sociobiology 41, 385-398.

[23] Collevatti, R.G., Campos, L.A.O. and Schoereder, J.H. (1997) 'Foraging behavior of bee pollinators on the tropical weed Triumfetta semitriloba: departure rules from flower patches', Insectes Sociaux 44, 345-352.

[24] Waddington, K.D., Nelson, C.M. and Page, Jr, R-E. (1998) 'Effects of pollen quality and genotype on the dance of foraging honeybees', Animal Behavior 56, $35-39$.

[25] Williams, N.M. and Thompson, J.D. (1998) 'Trapline foraging by bumblebees: III. Temporal patterns of visitation and foraging success at single plants', Behavioral Ecology 9, 612-621.

[26] Camazine, S. and Sneyd, J. (1991) 'A model of collective nectar source by honey bees: self-organization through simple rules', Journal of Theoretical Biology 149, 547-571.

[27] Biesmeijer, J.C., van Nieuwstadt, M.G.L., Lukacs, S. and Sommeijer, M.J. (1998) 'The role of internal and external information in foraging decisions of melipona workers (hymenoptera: meliponinae)', Behavior Ecology Sociobiology 42, 107116.

[28] Seeley, T.D. and Visscher, P.K. (1988) 'Assessing the benefits of cooperation in honeybee foraging: search costs, forage quality, and competitive ability', Behavior Ecology Sociobiology 22, 229-237.

[29] Colorni, A., Dorigo, M. and Maniezzo, V. (1991) 'Distributed optimization by ant colonies'. In: F. Varela and P. Bourgine (eds) Proceedings of the First European Conference on Artificial Life, pp. 134-142 (Paris, Elsevier).

[30] Dorigo, M., Maniezzo, V. and Colorni, A. (1996) 'Ant system: optimization by a colony of cooperating agents', IEEE Transactions on Systems, Man and Cybernetics, Part B 26, 29-41.

[31] Dorigo, M., Di Caro, G. and Gambardella, L.M. (1999) 'Ant algorithms for discrete optimization', Artificial Life 5, 137-172.

[32] Dorigo, M., Di Caro, G. and Stutzle, T. (2000) 'Ant algorithms', Future Generation Computer Systems 16, v-vii.

[33] Dorigo, M. and Gambardella, L.M. (1997a) 'Ant colonies for the traveling salesman problem', BioSystems 43, 73-81.

[34] Dorigo, M. and Gambardella, L.M. (1997b) 'Ant colony system: a cooperative learning approach to the traveling salesman problem', IEEE Transactions on Evolutionary Computation 1, 53-66.

[35] Bullnheimer, B., Hartl, R.F. and Strauss, C. (1999) 'An improved ant system algorithm for the vehicle routing problem', Annals of Operations Research 89, 319-328.

[36] Bullnheimer, B., Hartl, R.F. and Strauss, C. (1999) 'Applying the ant system to the vehicle routing problem'. In: S. Voss, S. Martello, I.H. Osman and C. Roucairol (eds) Meta-Heuristics: Advances and Trends in Local Search Paradigms for Optimization, pp. 285-296 (Boston, Kluwer Academic Publishers).

[37] Lučić, P. and Teodorović, D. (2002) 'Transportation modeling: an artificial life approach'. In: Proceedings of the 14th IEEE 'International Conference on Tools with Artificial Intelligence, Washington, DC, 4-6 November, pp. 216-223 (Washington, Brussels, Tokyo, IEEE Computer Society). 
[38] Teodorović, D. and Lučić, P. (2003) 'Schedule synchronization in public transit by fuzzy ant system', European Journal of Operational Research, under review.

[39] Zadeh, L (1965) 'Fuzzy sets', Information and Control 8, 338-353.

[40] Zadeh, L. (1973) 'Outline of a new approach to the analysis of complex systems and decision processes', IEEE Transactions on Systems, Man and Cybernetics SMC-3, 28-44.

[41] Mendel, J.M. (1995) 'Fuzzy Logic Systems for Engineering: A Tutorial', Proceedings of the IEEE, 83, 345-377.

[42] Lučić, P. and Teodorović, D. (2001) 'Bee system: modeling combinatorial optimization transportation engineering problems by swarm intelligence'. In: Preprints of the TRISTAN IV Triennial Symposium on Transportation Analysis, Sao Miguel, Azores Islands, Portugal, June, pp. 441-445 (São Miguel, Universidade dos Agores).

[43] Lučić, P. and Teodorovic, D. (2003) 'Computing with bees: attacking complex transportation engineering problems', International Journal on Artificial Intelligence Tools, 12, 375-394.

[44] Pattnaik, S.B., Mohan, S. and Tom, V.M. (1998) 'Urban bus transit route network design using genetic algorithm', Journal of Transportation Engineering, ASCE 124, 368-375.

[45] Ito, T. (1999) 'A genetic algorithm approach to piping route path planning', Journal of Intelligent Manufacturing 10, 103-114.

[46] Jong, J.C. and Schonfeld, P. (2003) 'An evolutionary model for simultaneously optimizing three-dimensional highway alignments', Transportation Research, Part B 37, 107-128.

[47] Lučić, P. and Teodorovic, D. (1999) 'Multi-objective simulated annealing model for aircrew rostering problem', Transport Research, Part A 33, $19-45$.

[48] Wren, A. and Wren, D. (1995) 'A genetic algorithm for public transport driver scheduling', Computers, Operations Research 22, 101-110.

[49] Teodorovic, D. and Radivojevic, G. (2002) 'A fuzzy sets approach to the dynamic dial-a-ride problem', Fuzzy Sets and Systems 116, 23-33. 